Ann. Sci. forest., 1973, 30 (3), 367-373.

\title{
CONCLUSION : EXPRESSION DES RÉSULTATS EN VUE DE LEUR APPLICATION PRATIQUE
}

\section{J. VENET}

Ingénieur général du G. R. E. F.

Professeur au Centre de Nancy de l'E. N. G. R. E. F.

et Directeur du Laboratoire de Chaire de Technologie du C. N. R. F.

Pourquoi les problèmes présentés dans cette réunion intéressent-ils au plus haut point l'aménagiste, chargé d'orienter et d'organiser la production d'un massif forestier?

prévoir à long terme: détermination des objectifs - choix du

L'aménagiste doit

$$
\text { traitement - évaluation de la production }
$$

organiser à court terme $\left(\mathrm{DAA}^{(1)}=20\right.$ à 30 ans) : établissement

du programme des coupes et travaux, appuyé par un bilan financier.

L'aménagiste se trouve placé à un carrefour

sollicitations d'ordre technologique et économique

sujétions imposées par la station (et l'essence).

Le propriétaire ou le gestionnaire d'un massif sont rarement satisfaits par la situation existante :

- les essences existantes ont souvent une productivité (quantitative, sinon qualitative) inférieure à celles "d'essences-miracle" (du type Douglas ou Grandis),

- le traitement qui est appliqué (par exemple taillis sous futaie) conduit à une production insuffisante de bois d'œuvre et excessive de bois d'industrie,

- l'état actuel de la forêt n'est pas normal (trop de vieux bois, déséquilibre des classes d'âge, etc.),

etc.

L'aménagiste est donc presque toujours conduit vers une intervention active sur le massif forestier.

(1) DAA = durée d'application de l'aménagement. 
Quelles informations possède-t-il sur les objectifs souhaitables?

Les statistiques nationales font ressortir l'évolution de la consommation et conduisent à des pronostics quant à la consommation future. Mais l'évolution de l'industrie est parfois plus rapide que la croissance des peuplements forestiers.

Sur le plan local, les besoins actuels des industries existantes et ceux des usines nouvelles (ou prévues) font l'objet de bilans, qui permettent d'organiser la récolte et d'orienter, dans une certaine mesure, la production (exemple Coucy-Vénizel).

Les organismes de recherches renseignent le sylviculteur (de façon de plus en plus précise) sur la qualité des produits (donc sur leur utilisation et leur prix) qui peuvent être obtenus avec les divers types d'essences, dans les divers types de station.

Il deviendra possible de cataloguer les stations à Chêne de tranchage, à Hêtre de déroulage, etc. comme on distingue actuellement (assez facilement) les stations qui conviennent ou non à la culture du Douglas.

Cela évitera des erreurs graves, comme celle qui consiste à cultiver du Chêne ou du Hêtre dans des stations où ces essences ne peuvent pas donner des produits de qualité élevée.

Les organismes de recherches donnent à l'aménagiste des informations non seulement sur les aptitudes technologiques des essences, dans des conditions de station déterminées, mais sur le traitement à leur appliquer pour obtenir une certaine qualité technologique (distance de plantation, emploi ou non de fertilisants, organisation des éclaircies, élagage artificiel, etc.).

Rappelons au passage que l'objectif de la sylviculture n'est pas toujours (ou uniquement) la production et que l'aménagiste tient compte aussi des autres rôles de la forêt (cynégétique, loisirs, protection, etc.).

L'aménagiste est donc suffisamment informé pour lui permettre de décider ce qu'il voudrait faire, mais il lui faut aussi savoir ce qu'il peut faire compte tenu des conditions écologiques régnant dans le massif à aménager.

Le problème essentiel, pour l'aménagiste, est d'avoir une connaissance précise des potentialités du massif forestier.

Un massif forestier à aménager, cela représente parfois 150 à 200 hectares pour une forêt communale, 500 à 600 hectares pour une seule série d'une forêt domaniale (dont certaines ont plus de 25000 hectares).

Le problème déborde donc largement celui de la connaissance des stations.

Quels documents et informations l'aménagiste possède-t-il sur les massifs forestiers?

Les cartes et plans le renseignent sur la situation géographique du massif et (complétés par les photographies aériennes) sur le modelé du relief.

Les stations météorologiques les plus voisines (mais généralement situées hors forêt) lui donnent les caractéristiques moyennes et extrêmes du climat et l'informent de ses agressions possibles contre les peuplements forestiers. 
La visite rapide des massifs apporte des indications complémentaires sur les facteurs topographiques (pente, exposition, etc.).

Les inventaires (en plein ou statistiques), effectués par parcelles, donnent les caractéristiques chiffrées des peuplements, par essences ou par groupes d'essences (nombre de tiges, diamètre moyen, surface terrière, volume sur pied, etc.). ${ }^{(1)}$

Les inventaires statistiques peuvent être complétés par des observations sur certains éléments susceptibles de conduire à la connaissance des stations (observations floristiques ou pédologiques) ou par l'étude, sur échantillons, des qualités technologiques, actuelles et potentielles, des bois.

une bonne connaissance d'ensemble du massif et de l'orientation générale qui peut lui être donnée (à rapprocher des cartes d'aires naturelles et de zones de dispersion, etc.),

Ceci conduit à

un inventaire détaillé et chiffré des peuplements actuels avec quelques indications sommaires sur leur évolution probable, cet inventaire étant réalisé par unités administratives (souvent géométriques et arbitraires) nommées « parcelles ».

Mais l'aménagiste doit organiser l'avenir du massif forestier, ce qui le conduit souvent, à l'époque actuelle, à apporter des transformations importantes quant au choix des essences à cultiver, au traitement, aux règles de culture, etc.

Ces transformations doivent correspondre, bien sûr, à des objectifs économiques, connus et précis, mais elles doivent tenir compte, avant tout, des potentialités du massif forestier c'est-à-dire d'un ensemble de stations, parfois très diverses, de surface et de forme très variables, parfois groupées, mais parfois dispersées en mosaïques assez confuses, dont les limites sont souvent imprécises, s'il y a changement progressif de conditions ou si la station est en évolution rapide, etc.

Il y a rarement coïncidence entre les limites des parcelles actuelles, numérotées et bien matérialisées sur le terrain, et les limites des stations. On trouve souvent, dans une même parcelle, des stations profondément différentes.

La connaissance de la station : critères distinctifs, signes évolutifs, potentialités visà-vis des diverses essences, etc. est donc l'élément de base d'un aménagement de forêt.

Mais il y a en France plus de douze millions d'hectares de forêts, dont quatre millions soumises au régime forestier. Les forêts soumises et une partie des forêts privées ont déjà fait l'objet, dans le passé, d'études plus ou moins poussées quant aux facteurs de la production et souvent d'inventaires.

Mais, selon les informations officielles, 4 millions d'hectares de forêts françaises sont non productives ou non susceptibles d'exploitation régulière dans leur état actuel, 4 millions d'hectares sont traités en taillis ou en taillis sous futaie c'est-à-dire, en général, d'une production non satisfaisante, et 4 millions d'hectares sont constitués de futaies dont la production est généralement bonne.

(1) Dans le cas des inventaires par échantillonnage, ces caractéristiques peuvent être cartographiées. 
A cela s'ajoutent de nombreuses surfaces de friches ou de landes reboisables.

Le problème de la connaissance des stations est donc aussi un problème considérable quant aux surfaces à étudier, et un problème urgent.

La méthode d'étude des stations, applicable pour l'aménagiste forestier, doit donc être à la fois simple et rapide - et susceptible d'être mise en œuvre par le personnel de terrain dont nous disposons.

Je pense, personnellement, que cette étude est à faire en deux temps.

$\mathrm{Au}$ cours d'une période de conception et d'organisation (par l'Ingénieur) est dressée la liste des types de stations susceptibles d'être rencontrés dans le massif et de servir de support à l'un des objectifs souhaitables (par exemple un médiocre peuplement de Chêne rouvre sur Canche flexueuse susceptible d'être remplacé par une plantation de Douglas). Cette liste est complétée, pour chaque station, par l'énumération et la définition des critères à observer.

Vient ensuite la période d'exécution, c'est-à-dire de la récolte des informations par le personnel de terrain, en utilisant généralement une méthode statistique.

Nous signalerons une petite difficulté matérielle : l'inventaire statistique des peuplements (en particulier des peuplements d'essences feuillues) se fait en général en hiver (en l'absence des feuilles qui gênent pour l'exécution de certaines mesures dendrométriques) alors que les observations phytosociologiques sont à faire en général en présence de la flore (souvent à diverses époques de l'année). Il en est de même d'ailleurs pour l'étude pédologique de certains sols qui tantôt sont gorgés d'eau, tantôt desséchés et durcis. Cela nécessite la matérialisation, sur le terrain, des «points » d'inventaire pour permettre un ensemble d'observations.

Il est nécessaire, à notre avis, que les observations floristiques et pédologiques soient faites aux mêmes points que les études dendrométriques, certains résultats des unes et des autres étant intéressants à lier (par exemple la hauteur totale, la vigueur et la qualité du chêne à rapprocher de la présence de certains végétaux herbacés et servant aussi à caractériser certains sols).

C'est vers la confection de ce catalogue des stations, au sens de groupes écologiques, fruit du travail concerté des chercheurs, que devrait conduire la discussion à laquelle nous venons d'assister. La combinaison des indices « écologiques » et des indices « dendrométriques » doit permettre l'établissement de listes de critères, simples et codifiables, grâce auxquels, dans la phase de conception et d'organisation des études de station, l'aménagiste pourra dresser l'extrait des « cas » susceptibles d'être rencontrés dans le massif à aménager.

Il aura ensuite à former le personnel d'exécution (très compétent en matière de sylviculture, mais auquel il faut apprendre à faire les observations pédologiques et floristiques nécessaires) et la réalisation statistique de ces observations lui donnera à la fois la répartition de la surface du massif par catégories de stations-objectifs et leur localisation (donc une certaine cartographie de ces stations).

Nous ne pouvons détailler ici le travail ultérieur de l'aménagiste qui fait appel, certes, à la connaissance des stations, mais aussi à celle des essences (et des races) et de leur tem- 
pérament, et à celle du traitement, sur la production qualitative et quantitative, chacun de ces deux points nécessitant un cycle d'études complet.

La connaissance de la station, répétons-le, est indispensable à l'aménagiste, à la fois pour :

- prévoir à long terme en choisissant les essences-objectifs et en chiffrant ces objectifs (âge et diamètre d'exploitabilité, qualité recherchée) et en fixant le traitement à leur appliquer,

- organiser à court terme, pour une première période d'intervention, le programme des travaux et coupes à effectuer : régénération naturelle ou artificielle dans le sous-ensemble de parcelles classé en régénération, coupes sélectives et travaux dans le sous-ensemble en amélioration.

Mais cette connaissance et cette cartographie forestière des stations doit sortir du cadre de la recherche et conduire à des instruments de travail utilisables par les forestiers gestionnaires et par leur personnel.

Les réunions qui viennent d'avoir lieu montrent que cela est parfaitement possible.

\section{DISCUSSIONS ET INTERVENTIONS}

M. Lebrun affirme l'intérêt des cartes d'identité des stations et des tables de production.

Il souhaite cependant que les résultats de la Recherche soient fournis aux praticiens sous une forme aussi élaborée que possible et que, par exemple, les cartes d'identité des stations soient assorties de cartes de stations pour certains massifs importants.

Il insiste sur l'intérêt des renseignements concernant le volume optimum sur pied et le volume à enlever par éclaircie, dans les tables de production.

M. Chenal fait ressortir l'étroite symbiose qui règne entre la Recherche et les Praticiens : la première apportant des solutions aux problèmes posés par les seconds (régénération du Sapin sur sols à Fétuque, choix d'essences de reboisement, fertilisation, etc.) et, en revanche, les praticiens apportant aux chercheurs des problèmes à résoudre, des terrains d'expérience et la collaboration de leur personnel.

M. Martinot-Lagarde insiste sur l'importance des cartes d'identité des stations grâce auxquelles les gestionnaires pourront procéder, eux-mêmes, aux recueils des informations qui permettront aux Services Techniques de l'O.N.F., bien outillés, pour cela, de procéder alors aux cartographies nécessaires.

MM. Bonneau, Timbal et Viard apportent quelques précisions quant aux problèmes de cartes : M. BonneaU, par exemple, signale que le C.N.R.F., sans pouvoir lui-même établir de cartes, peut cependant donner aux praticiens une idée des « cas » susceptibles d'être rencontrés dans leurs massifs (ce qui pourrait peut-être permettre aux Services Techniques de l'O.N.F. d'établir alors des « cartes-échantillon »).

M. Marion, au nom de la forêt privée, remercie le C.N.R.F. des journées d'études qu'il a organisées et de sa collaboration à l'amélioration des connaissances et de la produc- 
tion et insiste sur l'importance des recherches fondamentales, surtout quand elles sont effectuées en équipes pluridisciplinaires (comme c'est le cas au C.N.R.F.). Cette opinion va dans le même sens que celle qui avait été présentée par M. DeCOURT. M. MARION rappelle ensuite les besoins de la forêt privée en matière de recherches, en particulier en ce qui concerne la connaissance des stations.

M. Metro élargit le débat en insistant sur le rôle des experts internationaux et sur les besoins de ceux-ci en matière de recherche.

Il ressort de ce qui précède que les Praticiens souhaitent vivement la collaboration de la Recherche pour que puissent être établies, par exemple au cours des révisions d'aménagement, des cartes renseignées des massifs, relatives par exemple aux types de stations aux types de peuplements, aux objectifs.

Pour cela une connaissance de base est nécessaire : celle des caractéristiques actuelles et potentielles des stations, susceptibles d'être déterminées par des méthodes à la fois simples et sûres.

Ce type d'information devrait être rassemblé dans des fiches dites «cartes d'identité des stations » qui pourraient être réalisées, progressivement, par régions géographiques.

J. VENET

\section{UNE RÉFLEXION D'ENSEMBLE SUR LE COLLOQUE}

\section{J. Guillard}

Directeur du centre de Nancy de l'École nationale du génie rural des Eaux et des Forêts

Dans la succession des écosystèmes emboîtés les uns dans les autres (de l'écorce avec ses lichens jusqu'au massif forestier et au-delà) les chercheurs forestiers ont toujours privilégié à des fins d'analyse une coupure au niveau de la station, surface et ensemble relativement homogène en fonction de l'arbre et assez facile à saisir par des éléments simples de l'écotope et par la phytosociologie. Dans la mosaïque des stations, les forestiers gestionnaires ont pratiqué un découpage opérationnel la parcelle (à l'intérieur d'une station ou d'un groupe de stations affines).

Selon la discipline scientifique et le niveau des investigations utile ou nécessaire, le chercheur descend bien au-dessous de la taille de la station ou va bien au-delà. Mais il devrait toujours chercher à traduire la partie applicable de ses résultats au niveau de la station, le gestionnaire devant alors prendre le risque de transposition, d'agrégation ou de schématisation qui lui est imposé par les conditions de son métier.

Le forestier gestionnaire n'a de pouvoir efficace que sur une partie des facteurs internes de la station, sol et humus, microclimat, biocœnose, tandis que la modification volontaire des facteurs primaires lui est encore impossible.

La modification des écosystèmes internes à la station est sa tâche et sa responsabilité, il doit les modifier à son profit, en sauvegarder l'équilibre. C'est pour cela que toute lumière: 
sur leur fonctionnement lui est précieuse. Le chercheur universitaire apportera peut-être des vues sur l'énergétique des écosystèmes d'un certain niveau, le chercheur forestier devra en tirer les conséquences en matière de biomasse, le forestier gestionnaire parlera en tonnes de matière sèche ou encore en mètres cube de bois.

Actuellement, dans la grande majorité des cas, le « gestionnaire » essaye de faire évoluer l'écosystème qui correspond à la station dans la pénombre offerte par l'empirisme et l'expérience. Tout rayon de lumière venant illuminer tout ou partie du fonctionnement de cette boîte d'ombre lui permettra de mieux comprendre son action et de l'améliorer. De la physiologie de l'arbre à la physiologie du peuplement, beaucoup de connaissances sont encore indispensables. Toute amélioration de la connaissance des processus, des relations causales, des conséquences des actions lui permettra d'artificialiser de façon plus sûre et plus économique l'écosystème dont il est responsable, quel que soit le but recherché.

Le problème est de savoir non seulement ce qu'il est le plus urgent de connaître mais surtout de ce qu'il est le plus important ou le plus rentable de chercher. 